\title{
BYOD Secured Solution Framework
}

\author{
Md Iman Ali, Sukhkirandeep Kaur
}

\begin{abstract}
This research focuses on Secured design architecture of BYOD solution. Bring your Own Device is a project driven in most of the enterprises to provide internet access to employee personal devices and Guest users. It has Resulted in a trend to give Access to Employees Smart phones, tablets and personal to Improve employee productivity and address changing working preferences in todays digital age, The Enterprises are Benefiting by providing lesser Devices to their Employees, this is one of the major reasons for the Enterprise to invest in BYOD Infrastructure. Slowly BYOD becomes a rule rather exception. The BYOD Infrastructure provides their Employees an access to the Internet while being a trusted user accessing the Enterprise infrastructure, which is intended to be Secure. The BYOD Infrastructure also provides Employees to grant Guest User Internet Access to their Visiting Partners as well. Connecting external devices in Corporate network increases the cyber security risk and data leakage incident. Using BYOD services users can do malicious activities, try to gain unauthorized access to internal network which can lead into major security breach. Providing Internet access to the external devices using internal corporate network has resulted in a challenge in addressing significant Security risk, data theft and Shadow IT because of unsecured design architecture that compromise the security policy. Installing malware in BYOD and connecting to Internet network can also lead into serious damage and major security risk. A major concern is also to revoke internet access while employee is no more in the system. Incase if employee left and access does not get revoked on time also can be a risk. Poor BYOD design significantly lead into organization Cyber security risk. The Research Paper has Demonstrated the Use Case for Trusted User access and Untrusted Guest User access and Monitoring of the $B Y O D-U s e r$ activity and Audit compliance. In this research paper, a secured Design Architecture, Implementation and analysis is conducted. A practical analysis is conducted regarding the design and a Secure BYOD model is demonstrated. This study also highlights the Secured model of BYOD implementation maintaining organization laid down security policy so that enterprise CEO, CIO, CISO can address concerns for securing the Enterprises BYOD Infrastructure.
\end{abstract}

Keywords-BYOD, Security. BYOD framework

\section{INTRODUCTION}

With the growing rate of Internet access required in working environment on employee's personal devices, the phenomena called as BYOD. Bring your own device in the organization and providing internet access to personal devices become primary requirement for the organization IT department. BYOD becomes rule rather exception over the time. BYOD solution which is used in almost all the organizations, universities, institutions. BYOD provide the

Revised Manuscript Received on October 30, 2019.

* Correspondence Author

Md Iman Ali*, Computer Application, LPU/Punjab, India

Dr SukhkiranDeep Kaur, CSE, LPU/Punjab, India

(C) The Authors. Published by Blue Eyes Intelligence Engineering and Sciences Publication (BEIESP). This is an open access article under the CC BY-NC-ND license (http://creativecommons.org/licenses/by-nc-nd/4.0/) internet access to personal devices like mobile, tablet and other internet devices. Bring your own device in the organization provide the extra luxury facility for employee satisfaction and need to seamless internet access within the organization boundary.

BYOD services involved very high security risk and threats to the organization. Allowing internet access to employees personal devices can lead into potential security risk, data leakage, loss of control if proper security mechanism is not in place[1]. Using mobile devices for personal and professional work itself has security risk[2].Specially design architecture of BYOD is very critical in nature, ignorance on BYOD design can lead into a major security and organizational potential data loss. Then how the BYOD should be design ? in an organization there can be 2 different set of BYOD users like employee which an active directory user and guest wifi access to the users who are not active directory users, untrusted users. How this 2 types of users can be segregated and create secure solution of BYOD ? This study purely focus on the design architecture and analysis of the security parameter and how to create two different security architecture using the same wireless architecture. Finally after test and analysis this study suggest a framework of creating a secure model of BYOD architecture for corporate wireless, Employee personal devices access and Guest user access.

This study also analyse a new and highlight the security risk of using traditional BYOD services to the cloud data which is organization private cloud data which is new study area. But data protection mechanism is also crucial part while giving internet access to the personal devices using same Wireless Access point and wireless controller. While implementing BYOD solution organization need to maximize the advantage but minimize the risk[3]. Organization need to be aware of security risk while designing and implementing the BYOD solutions. Since same Wireless access point need to be used in corporate wireless services and BYOD wireless service. Employee satisfaction of an organization is measured with quality of work culture and facility to the employee. As internet becomes one of the fundamental component of for the employee, now a days. Every employee of an organization has minimum of $2 / 3$ devices at all the time, Official and personal. BYOD market About to hit $\$ 367$ billion by 2022, up from just $\$ 30$ billion in 2014 (Source: BetaNews). While in official Laptop employee gets internet services and security policies are defined in the perimeter firewall and all the web gateways. But every employee demands internet services on their personal devices.

Giving Internet access in personal device using the same wireless Access Point and the controller traffic segregation normally done with SSID, layer 3 authentication etc.

While giving internet access to their personal devices as the concept called as "Bring your own device". BYOD design has certain limitations. 


\section{DESIGN METHODOLOGY FRAMEWORK}

Proposed BYOD Secured model for Employee own personal device and Guest personal devices are both segregated design.Design and access method of three different segment of architecture using same wireless access point and wireless controller along with AAA server and user database are shown in 3 different figure.

\section{A CASE-1: BYOD-Trusted-User: AD user}

In this scenario BYOD user is the user who is an employee of the organization and user id is retrieved from active directory domain controller. User authentication mechanism is based on the existence of the user id in Active directory employee database. Block diagram Architecture for BYODTrusted-Employee devices having userbase in organization active directory.

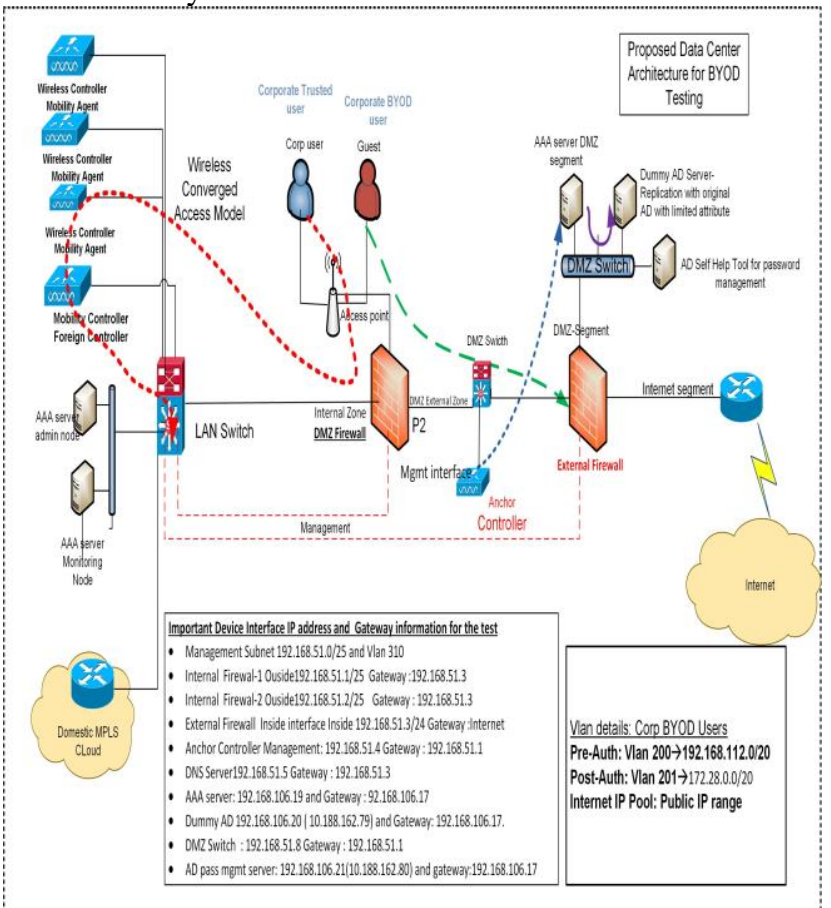

Fig-1: Proposed Architecture Block diagram for the test for the user who are active users in active directory.

\section{B TESTING COMPONENTS USED FOR THE DEMONSTRATION}

Testing has been done with below components products with their make and model.

\begin{tabular}{|l|l|l|}
\hline Seq\# & Technology/Service & Product used for test \\
\hline 1 & AAA server & $\begin{array}{l}\text { Cisco Identity Service } \\
\text { Engine }\end{array}$ \\
\hline 2. & Internal Firewall & Cisco Firewall \\
\hline 3. & External Firewall & Checkpoint Firewall \\
\hline 4. & Anchor Controller & Cisco WLC 5508 \\
\hline 4. & $\begin{array}{l}\text { Foreign controller/Mobility } \\
\text { controller }\end{array}$ & Cisco WLC \\
\hline 5. & Mobility Agent & Cisco 3850 \\
\hline 6. & Active directory & Microsoft AD \\
\hline 7. & Router & Cisco Router \\
\hline 8. & BYOD devices & Android/Iphone \\
\hline 9 & Corporate Device & Laptop \\
\hline
\end{tabular}

Table-1: Products/devices used during the test.

\section{2.c Traffic flow for BYOD architecture during the test}

Traffic flow of the BYOD-Trusted-User having user entry in Active Directory

\begin{tabular}{|l|l|l|l|}
\hline Seq\# & Source & Destination & $\begin{array}{l}\text { TCT/UDP } \\
\text { Port }\end{array}$ \\
\hline 1 & $\begin{array}{l}\text { Foreign } \\
\text { Controller }\end{array}$ & AAA server & 1812 \\
\hline 2. & BYOD user & DNS & 53 \\
\hline 3. & BYOD user & AAA server & 8443 \\
\hline 4. & BYOD & AAA server & 8905 \\
\hline
\end{tabular}

Table-2: Traffic flow with port numbers

1st packet of the BYOD device is towards AAA server on port 1812 as authentication request but this is proxied through Foreign controller, post user gets Pre-auth IP address from External Firewall

As shown in Fig-1 BYOD device directly land up in External Firewall segment bypassing the corporate network.

This user traffic is not seen anywhere in the corporate network even though same access point is used.

Security risk[4] and policy compliance of the organization and protection of core infrastructure from BYOD device is maintained with this approach. Implementing BYOD crashes will Security breach[5] and will be bigger impact to organization data.

While BYOD device will connect the SSID "BYODEmployee" will get pre-auth IP which does not have any access apart from reachability of the AAA server and DNS.

2nd Packet will goto DNS for resolution of the Certificate name.

In this study authentication method is PKI used as Certificate based authentication which is most secured and encrypted[6] method of authentication of 802.1x. Purely layer 2 model of authentication has been used. In this test this is configured and tested until certificate based authentication happened user will not get the Post auth IP.

\section{C.1 TRAFFIC FLOW CHART}

Flow of authentication as mentioned below

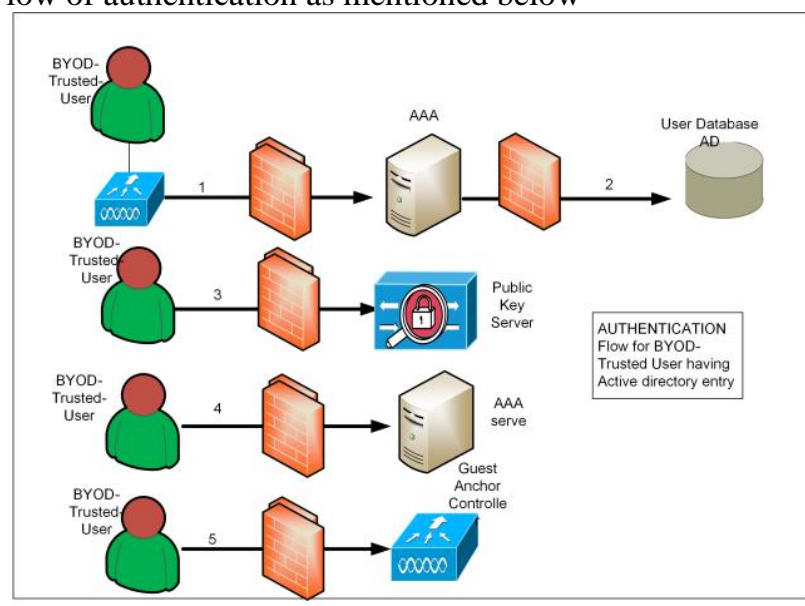

Fig-2: Step by step Traffic flow this demonstration As shown in fig-2 when BYOD user request for authentication with a proxy of wireless Lan controller, request goes to AAA server (1) and then AAA server request to User data base (2) back to back to authenticate valid user and active user. Post AD respond back to AAA with response as "Valid", it redirects for certificate and here the main point for $802.1 \mathrm{x}$ authentication mechanism to be used which is secured[7] model.

Published By:

Blue Eyes Intelligence Engineering \& Sciences Publication

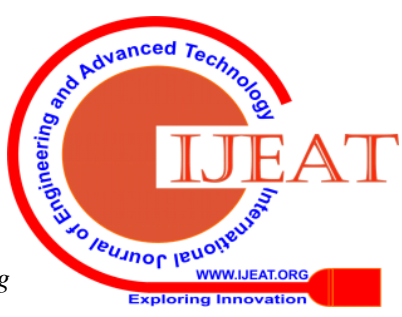


In this phase BYOD device gets the certificate (5). Then AAA server response back to Anchor controller to change the Vlan, which is called Post auth Vlan (Internet access IP).

Post-auth Network segment will be having access of Internet. This policy is on external Firewall only as below

\begin{tabular}{|l|l|l|l|l|}
\hline Seq & Source & Destination & Port & Natting \\
\hline 1 & $\begin{array}{l}\text { Post-Auth } \\
\text { Vlan }\end{array}$ & Any & Any & Yes \\
\hline
\end{tabular}

Table-1: Firewall policy for internet access

In this stage BYOD-Trusted Employee will get internet access without any IP routing of this Network in corporate internal network. The major security concern of connecting personal device[8] in corporate network is addressed in segregating the BYOD Segment and with certificate based secured authentication. In this case security posture[9] and associated risk are also addressed because vulnerable device can be major security threats. BYOD design should comply security policies of the enterprise[10]. In this test this is completely segregated from corporate network.

\section{RESULT AND ANALYSIS}

Test result and analysis of the BYOD-Trusted-Users with EAP-TLS secured certificate based authentication Steps during authentication, logs from AAA server, this test is done with the BYOD device MAC address: 94:65:2D:E8:A4:89

\begin{tabular}{|l|l|}
\hline Event \# & Event Details \\
\hline 11001 & Received RADIUS Access-Request \\
\hline 11017 & RADIUS created a new session \\
\hline 15049 & Evaluating Policy Group \\
\hline 15008 & Evaluating Service Selection Policy \\
\hline 11507 & Extracted EAP-Response/Identity \\
\hline 12500 & Prepared EAP-Request proposing EAP-TLS with chal \\
\hline 12625 & Valid EAP-Key-Name attribute received \\
\hline 11006 & Returned RADIUS Access-Challenge \\
\hline 11001 & Received RADIUS Access-Request \\
\hline 11018 & RADIUS is re-using an existing session \\
\hline 12502 & Extracted EAP-Response containing EAP-TLS challen \\
\hline 12800 & Excepting EAP-TLS as negotiated \\
\hline 12805 & Extracted TLS ClientHello message \\
\hline 12806 & Prepared TLS ServerHello message \\
\hline 12807 & Prepared TLS Certificate message \\
\hline 12808 & Prepared TLS ServerKeyExchange message \\
\hline 12809 & Prepared TLS CertificateRequest message \\
\hline 12505 & Prepared EAP-Request with another EAP-TLS challen \\
\hline 11006 & Returned RADIUS Access-Challenge \\
\hline 11001 & Received RADIUS Access-Request \\
\hline 11018 & RADIUS is re-using an existing session \\
\hline 12504 & Extracted EAP-Response containing EAP-TLS challen \\
\hline 12505 & Prepared EAP-Request with another EAP-TLS challen \\
\hline 11006 & Returned RADIUS Access-Challenge \\
\hline 11001 & Received RADIUS Access-Request \\
\hline 11018 & RADIUS is re-using an existing session \\
\hline 12504 & Extracted EAP-Response containing EAP-TLS challen \\
\hline 12505 & Prepared EAP-Request with another EAP-TLS challen \\
\hline 11006 & Returned RADIUS Access-Challenge \\
\hline 11001 & Received RADIUS Access-Request \\
\hline 11018 & RADIUS is re-using an existing session \\
\hline 12504 & Extracted EAP-Response containing EAP-TLS challen \\
\hline 12505 & Prepared EAP-Request with another EAP-TLS challen \\
\hline 11006 & Returned RADIUS Access-Challenge \\
\hline 11001 & Received RADIUS Access-Request \\
\hline 11018 & RADIUS is re-using an existing session \\
\hline 12504 & Extracted EAP-Response containing EAP-TLS challen \\
\hline 12505 & Prepared EAP-Request with another EAP-TLS challen \\
\hline
\end{tabular}

\begin{tabular}{|c|c|}
\hline 11006 & Returned RADIUS Access-Challenge \\
\hline 11001 & Received RADIUS Access-Request \\
\hline 11018 & RADIUS is re-using an existing session \\
\hline 12504 & Extracted EAP-Response containing EAP-TLS challen \\
\hline 12505 & Prepared EAP-Request with another EAP-TLS challen \\
\hline 11006 & Returned RADIUS Access-Challenge \\
\hline 11001 & Received RADIUS Access-Request \\
\hline 11018 & RADIUS is re-using an existing session \\
\hline 12504 & Extracted EAP-Response containing EAP-TLS challen \\
\hline 12568 & $\begin{array}{l}\text { Lookup user certificate status in OCSP cache - certific } \\
\text { Services Endpoint Sub CA - AAA1 }\end{array}$ \\
\hline 12570 & $\begin{array}{l}\text { Lookup user certificate status in OCSP cache succee } \\
\text { Certificate Services Endpoint Sub CA - AAA1 }\end{array}$ \\
\hline 12554 & $\begin{array}{l}\text { OCSP status of user certificate is good - certificate for } \\
\text { Endpo int Sub CA - AAA1 }\end{array}$ \\
\hline 12568 & $\begin{array}{l}\text { Lookup user certificate status in OCSP cache - certific } \\
\text { Services Node CA - AAA01 }\end{array}$ \\
\hline 12570 & $\begin{array}{l}\text { Lookup user certificate status in OCSP cache succee } \\
\text { Certificate Services Node CA - AAA01 }\end{array}$ \\
\hline 12554 & $\begin{array}{l}\text { OCSP status of user certificate is good - certificate for } \\
\text { Node CA -AAA01 }\end{array}$ \\
\hline 12835 & Expired certificate was accepted from the client \\
\hline 12811 & Extracted TLS Certificate message containing client c \\
\hline 12812 & Extracted TLS ClientKeyExchange message \\
\hline 12813 & Extracted TLS CertificateVerify message \\
\hline 12804 & Extracted TLS Finished message \\
\hline 12801 & Prepared TLS ChangeCipherSpec message \\
\hline 12802 & Prepared TLS Finished message \\
\hline 12816 & TLS handshake succeeded \\
\hline 12509 & EAP-TLS full handshake finished successfully \\
\hline 12505 & Prepared EAP-Request with another EAP-TLS challen \\
\hline 11006 & Returned RADIUS Access-Challenge \\
\hline 11001 & Received RADIUS Access-Request \\
\hline 11018 & RADIUS is re-using an existing session \\
\hline 12504 & Extracted EAP-Response containing EAP-TLS challen \\
\hline 61025 & Open secure connection with TLS peer \\
\hline 15041 & Evaluating Identity Policy \\
\hline 15048 & Queried PIP - Normalised Radius.RadiusFlowType \\
\hline 22072 & Selected identity source sequence - BYOD_Sequence \\
\hline 22070 & Identity name is taken from certificate attribute \\
\hline 22037 & Authentication Passed \\
\hline 12506 & EAP-TLS authentication succeeded \\
\hline 24423 & ISE has not been able to confirm previous successful \\
\hline 15036 & Evaluating Authorization Policy \\
\hline 15048 & Queried PIP - Cisco.cisco-av-pair \\
\hline 15048 & Queried PIP - Network Access.NetworkDeviceName \\
\hline 15048 & Queried PIP - Radius.NAS-Port-Type \\
\hline 15048 & Queried PIP - EndPoints.LogicalProfile \\
\hline 15048 & Queried PIP - EndPoints.BYODRegistration \\
\hline 15048 & Queried PIP - Network Access.EapAuthentication \\
\hline 15048 & Queried PIP - Network Access.UseCase \\
\hline 15048 & Queried PIP - CERTIFICATE.Subject Alternative Nam \\
\hline 15048 & Queried PIP - Radius.Calling-Station-ID \\
\hline 15016 & Selected Authorization Profile - Change Vlan \\
\hline 22081 & Max sessions policy passed \\
\hline 22080 & New accounting session created in Session cache \\
\hline 11503 & Prepared EAP-Success \\
\hline 11002 & Returned RADIUS Access-Accept \\
\hline
\end{tabular}

Table -3: Table shows the sequential events during the authentication process.

In this state BYOD-Trusted-Employee user is accepted after completion of the authentication and the devices COA(Change of authorization) triggered. Post COA the device will get Post-auth IP address from isolated network, which will directly go to the internet through external Firewall. Implication and security risk even after this isolated environment is if the External Network Internet is used as the same Internet exit path of the enterprise. 
If the enterprise has APNIC IP address registered then any malicious activity done by the BYOD user will be the responsibility of the enterprise. This study highlight and suggest to use a different Internet exit path apart from corporate Internet.

The 2nd risk also can occur with Cloud services if corporate IP is used for BYOD user, The ideal case of any enterprise cloud services is creating NSG in cloud for accepting traffic from enterprise Public IP segment. Incase if this use same IP segment then BYOD users will get access to the enterprise trusted infrastructure hosted in cloud. So this study explored the risk of cloud services so suggest to use different Public IP/separate isolated public network to build a secure infrastructure.

\section{A Monitoring of the BYOD-User activity and Audit compliance}

Monitoring of the activities using Public IP as per the IP address retention and use Policy, Lawful disclosure[11] for services provider is mandate to manage the logs. In the case of any malicious activities logs has to be maintained incase of any dispute and investigation situation Service provider will directly identify the IP assigned organization. In this case user identification will be the responsibility of the enterprise while ISP will only identify the enterprise where Public IP released. User acceptance policy also need to be agreed and signed by the BYOD user as per organization security policy framework measurement.[4]

Monitoring of the BYOD users activity is a major concern, so BYOD solution should have proper log management model to capture logs and identification of the users, and storing such large amount of logs.

In this research Cisco Identity service[12] solution is used in connection with additional user database as active directory used while Aruba CPPM Clearpass Policy manager[13] solution is also one the solution to manage logs. ARUBA CPPM which is also use for the IOT, Mobile devices and access management, which works in role base access model. But either Cisco or Aruba both solution does not comply the enterprise security and audit requirement, which is enterprise responsibility. So this study address the complete end to end requirement while enabling BYOD and maintain security compliance.

\section{B CASE-2: BYOD-Untrusted-User: Not active Directory user}

The use case of this type of Wireless access is generally provided to untrusted users who are normally visitor to the organization. In such case user id created using Lobby administrator portal, or self sign in method which is normally a AAA server use model. In this case Guest user id get created with some time duration or permanent duration. This situation can be addresses if AAA server is local to the branch. But security challenges comes when AAA server is not local.

3.C CASE-3: BYOD-Untrusted-User: Not active Directory user and user is located in Remote Branch and AAA server is centralized.

The design of the BYOD architecture impact to cost. The major cost of the BYOD are Hardware,[14] software, Bandwidth, Network and Operation.

Different deployment model approach cost are different. While CIO of the organization initiate the project, always cost benefit analysis happened and design approach also get optimized. One of the cost saving model is centralized architecture and in this test AAA server is placed in Centralized location and BYOD-untrusted user located in remote Branch over MPLS. This use case is tested to save the cost of the deployment and overall project cost.

\section{DISCUSSION}

This research study and design framework has a major advantage for the BYOD-Trusted-Employee as if any employee travel across any other Branch office of the organization will have seamless access of BYOD internet services, as certificate based authentication is used and seamless onboarding process and secured method of BYOD access. SSID has to be same and same AAA is used and central method of authentication even this can be in distributed architecture.

\section{A. Contribution}

Main contribution of this study after analyzing the traffic routing requirement from BYOD users devices to the AAA server is how BYOD design architecture should be to reduce security risk of the organization. BYOD-Trusted-Users who are basically active directory users authentication model, authentication mechanism and onboarding process without any risk of the organization laid down security framework. 2nd type of the users BYOD-untrusted-users who are not organization active directory users are the biggest challenging onboarding, which need to be taken care with additional security parameter. In the 2nd case of BYOD users authentication ideally recommended to have easy onboarding process but with additional security measure. The risk of onboarding 2nd category BYOD user is their authentication mechanism, in such situation initially devices gets the IP address which is pre-auth IP, in-order to authenticate this devices, Pre-auth IP need to have IP reachability to the AAA server or controller should have proxy the traffic to AAA server. The organization recommended secured design model suggest devices pre-auth IP should be directly to external network, but in this case authentication request does not proxied to AAA server which is in trusted zone. Guest user pre-auth traffic should not be routed to design secure infra.

Process for Authentication as

When BYODE AR $\rightarrow$ AAA

$$
\text { If BYOD User==AD }
$$

Then $\mathrm{ADR}=$ Success else $\mathrm{ADR}=$ False

IF ADR $=$ True then AAASR $\rightarrow$ WLC for Post-auth.

To overcome such situation and reduce the risk of routing untrusted IP network in trusted MPLS/LAN zone, external encrypted tunneled to be created from Branch office Untrusted Guest Internet zone to Data center Guest internet zone.Internet Access revoke from BYOD also one of the major attention point. In this solution this is been tested while the employee gets deactivated from Active directory, then certificate gets expired and reauthentication request get failed thus security compliance is maintained 3rd category of the corporate wireless users does not have any such challenges as their wireless Controller and AAA servers resides in trusted Network and same Wireless Access points are used.

This study contributes to create a secured framework of the BYOD implementation in centralized and distributed model. While keeping all organization security policy.

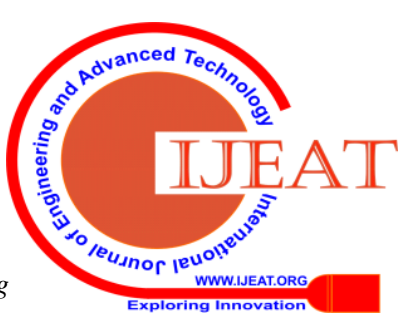




\section{B. Implication}

This study highlight the BYOD design that Cost factor which is one of the major direction before adopting the BYOD solution. Since this service is an employee satisfaction category service in work environment so every CIO of the organization looks for reducing the cost. But in saving the cost major risk can get involved in security compliance.

Selecting the BYOD design depends on the organization security framework strength

\section{Future study}

This study explored and suggest that more analysis required in BYOD environment in connection of Cloud Security. In Traditional BYOD design does not have cloud security analyses. But since cloud adoption is exponentially growing and BYOD traffic exits from same gateway in ideal case, so BYOD design and cloud security with Software define WAN need to studied in depth

\section{CONCLUSION}

Organization need to address the security risk while giving the internet access to employee on their personal devices from corporate wireless infrastructure.

It is not possible to segregate wireless access point and wireless Lan controller for Corporate intranet wireless and BYOD wireless in same physical area. Co-channel interference and adjacent channel interference lead into major interruption of services in RF Media. So in this study multiple SSID broadcasted in same area using same AP, Controller for BYOD services in secured way. While giving the internet access using same controller and Access point, security model must be designed in such a way so that end to end traffic segregation is maintained .

In this study, it is demonstrated that BYOD service to be divided as Two different profiles.

Firstly for BYOD corporate user profile which is a Trusted User Profile ,It is demonstrated to be a certificate based authentication which can be recommended as secured model. and Secondly for guest access which is Untrusted Profile, it is recommended to have Layer three open authentication.

In either of the case it is recommended to have the gateway of the BYOD devices outside the corporate LAN infrastructure, which can be considered as best secured way of managing BYOD infrastructure. Even if the personal devices are connected to the same Wireless Access point but the gateway of the BYOD devices should be outside the DMZ Firewall as referred in Figure 1 which Segregates LAN and External Segment and maintain secured way of access.

This study demonstrated a very secured model of traffic flow of BYOD , before even getting the Pre-authentication IP address, traffic is encrypted with EOIP tunnel and landed directly outside corporate LAN. In this scenario this is analysed that BYOD (Both type of SSID) vlan does not even required to be created in corporate Core LAN infrastructure. even there is no requirement of Routing of those BYOD IP segment inside LAN. This makes Corporate LAN infrastructure secured while giving internet access to BYOD user.

BYOD access management and revoking of Internet access as an exit management process which is very large numbers in bigger organization also becomes automatic with this mechanism of BYOD architecture, which protect organization from security risk and becomes system driven process rather manual intervention.
This study also analyse one important direction of designing the BYOD and cloud security architecture and risk and mitigation towards today's demand cloud services adoption.

\section{REFERENCES}

1. Z. Mitrovic, I. Veljkovic, G. Whyte, and K. Thompson, "Introducing BYOD in an organisation: the risk and customer services viewpoints," p. 26, 2014

2. P. K. Gajar, A. Ghosh, and S. Rai, "BRING YOUR OWN DEVICE (BYOD): SECURITY RISKS AND MITIGATING STRATEGIES," p. 9, 2010.

3. C. Walker-Osborn, S. Mann, and V. Mann, "to Byod or ... not to Byod," ITNOW, vol. 55, no. 1, pp. 38-39, Mar. 2013.

4. C. Z. Tu, J. Adkins, and G. Y. Zhao, "Complying with BYOD Security Policies: A Moderation Model Based on Protection Motivation Theory," Journal of the Midwest Association for Information Systems, vol. 2019, no. 1, p. 19, 2019.

5. P. Baillette, Y. Barlette, and A. Leclercq-Vandelannoitte, "Bring your own device in organizations: Extending the reversed IT adoption logic to security paradoxes for CEOs and end users," International Journal of Information Management, vol. 43, pp. 76-84, Dec. 2018.

6. J. Allen and J. Wilson, "Securing a Wireless Network," p. 3

7. L. Maccari, R. Fantacci, T. Pecorella, and F. Frosali, "Secure, fast handhoff techniques for $802.1 \mathrm{X}$ based wireless network," in 2006 IEEE International Conference on Communications, Istanbul, 2006 pp. 3917-3922.

8. K. W. Miller, J. Voas, and G. F. Hurlburt, "BYOD: Security and Privacy Considerations," IT Professional, vol. 14, no. 5, pp. 53-55, Sep. 2012.

9. [M. Ph. Stoecklin et al., "Passive security intelligence to analyze the security risks of mobile/BYOD activities," IBM Journal of Research and Development, vol. 60, no. 4, pp. 9:1-9:13, Jul. 2016.

10. Yong Wang, Jinpeng Wei, and K. Vangury, "Bring your own device security issues and challenges," in 2014 IEEE 11th Consumer Communications and Networking Conference (CCNC), Las Vegas, NV, 2014, pp. 80-85.

11. P. Iyengar, "IP ADDRESSES AND EXPEDITIOUS DISCLOSURE OF IDENTITY IN INDIA," vol. 9, p. 28.

12. S. Jose, "Cisco Identity Services Engine Administrator Guide, Release 2.2," p. 1240.

13. P. Kaspian, "ARUBA CLEARPASS NETWORK ACCESS CONTROL,”p. 5.

14. J. Loucks, R. Medcalf, L. Buckalew, and F. Faria, “An astounding 89 percent of companies are enabling their employees to use their own devices for work purposes.," Economic Analysis, p. 26

\section{AUTHORS PROFILE}

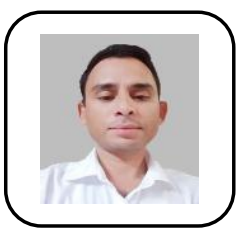

Md Iman Ali is a Research scholar, in the department of computer application at Lovely Professional University, Punjab, India. $\mathrm{He}$ has completed MCA and DOEACC "A" level. As a professional certification he is CCIE Lab certified. His research interest area include BYOD secured solutions, Cyber security, SD-WAN Security, Cloud Security and Smart city Cyber Forensic.

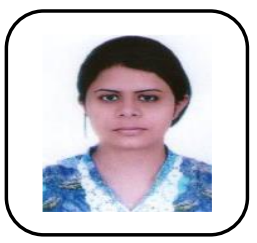

Dr Sukhkiran deep Kaur, is an assistant professor in LPU, Punjab, India. She has received $\mathrm{PhD}$ from NIT, Srinagar, India. Her current research interest includes Cyber Security, BYOD solutions, Forensic Science. 